\title{
LINEAR SYZYGIES, FLAG COMPLEXES, AND REGULARITY
}

\author{
ALEXANDRU CONSTANTINESCU, THOMAS KAHLE, AND MATTEO VARBARO
}

\begin{abstract}
We show that for every $r \in \mathbb{Z}_{>0}$ there exist monomial ideals generated in degree two, with linear syzygies, and regularity of the quotient equal to $r$. Such examples can not be found among Gorenstein ideals since the regularity of their quotients is at most four. We also show that for most monomial ideals generated in degree two and with linear syzygies the regularity is $O(\log (\log (n))$, where $n$ is the number of variables.
\end{abstract}

Let $n$ be a positive integer, $S=K\left[x_{1}, \ldots, x_{n}\right]$ the polynomial ring in $n$ variables over a field $K$. Any quotient $S / I$ by some homogeneous ideal $I \subseteq S$ has a minimal graded free resolution. The number of minimal generators of a given degree of the free modules occurring in the resolution are independent of the resolution chosen and define the Betti numbers $\beta_{i, j}(S / I)$. The Castelnuovo-Mumford regularity of $S / I$ is $\operatorname{reg}(S / I):=\max \left\{j-i: \beta_{i, j} \neq 0\right\}$.

Bounding the regularity. Equipped with these definitions it is a basic question to understand extreme values and shapes of the Betti numbers and the modules that realize them. One line of research, which we contribute to here, is to bound the regularity in terms of the number of variables for specified classes of ideals. To get interesting bounds one has to put strong restrictions. A class of examples due to Mayr and Meyer shows that even for quadratically generated binomial ideals in $n$ variables, regularity of the order of $2^{2^{n}}$ is possible MM82, BS88. In view of this result, interest shifted to specific classes of ideals with good geometric or algebraic properties. For example, Eisenbud and Goto conjectured in EG84 that if $I$ is a prime ideal defining a variety of codimension $r$ and degree $d$ in $\mathbb{P}^{n-1}$, then $\operatorname{reg}(S / I) \leq d-r$. See BM93 for a broader overview. In other directions, the defining ideals of Koszul algebras have a good bound: If $S / I$ is $\operatorname{Koszul}$, then $\operatorname{reg}(S / I) \leq n$. The same bound is also satisfied by monomial ideals generated in degree two. In order to get stricter bounds, one has to impose more restrictions on the class of ideals. In this note we are interested in quadratically generated ideals whose resolutions are linear for a few steps:

Definition 1. For any positive integer $p$, the $K$-algebra $S / I$ satisfies property $N_{p}$ if:

$$
\beta_{i, j}(S / I)=0 \quad \forall i \in\{1, \ldots, p\} \text { and } j \neq i+1 .
$$

If $S / I$ is Koszul and satisfies property $N_{p}$, a recent result of Avramov, Conca, and Iyengar [ACI13, Theorem 6.1] implies that

$$
\operatorname{reg}(S / I) \leq 2\lfloor n /(p+1)\rfloor+1 .
$$

It is currently unknown whether the above bound is sharp. However, ideals for which $S / I$ is Koszul, that satisfies property $N_{2}$, and have $\operatorname{reg}(S / I) \sim \sqrt{n}$ exist [ACI13, Example 6.9]. In

Date: October 2014.

2010 Mathematics Subject Classification. Primary: 13F55; Secondary: 13D02, 20F55. 
contrast to the belief that ideals defining Koszul rings and quadratic monomial ideals should have similar homological properties (they often do), if $I$ is a monomial ideal such that $S / I$ satisfies $N_{p}$, a much better bound has been established by Dao, Huneke, and Schweig.

Theorem 2. DHS13 Let $I \subseteq S=K\left[x_{1}, \ldots, x_{n}\right]$ be a monomial ideal such that $S / I$ satisfies $N_{p}$ for some $p \geq 2$. Then

$$
\operatorname{reg}(S / I)<\log _{(p+3) / 2}\left(\frac{2 n}{p}\right)+2 .
$$

Even if the above bound becomes stricter when $p$ grows, its logarithmic nature already shows for $p=2$. Thus, in a sense, the crucial assumption is that $S / I$ satisfies $N_{2}$. In other words, when $I$ is quadratically generated and its syzygy module is linear. For this reason, we mainly work in the case where $I$ is a monomial ideal such that $S / I$ satisfies $N_{2}$. Without loss of generality we deal with square-free monomial ideals, since by polarization we can always assume this (at most doubling the number of variables). Such ideals are known as edge ideals: Given a simple graph $G$ on $n$ vertices, its edge ideal is defined as

$$
I(G)=\left(x_{i} x_{j}:\{i, j\} \text { is an edge of } G\right) \subseteq S=K\left[x_{1}, \ldots, x_{n}\right] .
$$

It is often convenient to think of edge ideals as Stanley-Reisner ideals of flag simplicial complexes. Precisely, $I(G)=I_{\operatorname{In}(G)}$ where $\operatorname{In}(G)$ is the independence complex of $G$. By definition, every flag simplicial complex is of this form. From the explicit description of the syzygy module of a monomial ideal, one can immediately check that

$$
S / I(G) \text { satisfies } N_{2} \Longleftrightarrow \operatorname{In}(G) \text { has no induced 4-cycles. }
$$

More generally, it can be proved that $N_{p}$ is equivalent to $\operatorname{In}(G)$ having no induced $(p+2)$ cycles (cf. [DHS13, Theorem 2.7]). If $\Delta$ is a flag simplicial complex with no induced 4-cycles, we say that $\Delta$ is flag-no-square.

Remark 3. Flag-no-square simplicial complexes are called 5-large in the literature on hyperbolic Coxeter groups, such as [JŚ03]. More generally a flag complex is $k$-large if its systole - the shortest induced cycle - has length at least $k$. Confusingly, in that literature an induced subcomplex is called a full subcomplex (even if then what is arguably an empty cycle goes by the name of full cycle).

Our first result strengthens Theorem 2 in the case that $S / I$, besides satisfying $N_{2}$, is also Gorenstein. Then there is a universal bound for the regularity. We learned the averaging argument in its proof from Davis' book on Coxeter groups [Dav08, Lemma 6.11.5].

Theorem 4. Let $I \subseteq S$ be a monomial ideal such that $S / I$ is Gorenstein.

(i) If $S / I$ satisfies $N_{2}$, then $\operatorname{reg}(S / I) \leq 4$.

(ii) If $S / I$ satisfies $N_{3}$, then $\operatorname{reg}(S / I) \leq 2$.

Proof. Up to polarization, we can assume that $I=I_{\Delta}$ where $\Delta$ is a Gorenstein flag simplicial complex. By [Sta77, Theorem 7] there exist a Gorenstein* complex $\Gamma$ and a simplex $\tau$ such that $\Delta=\tau * \Gamma$. Thus, without loss of generality, we assume that $\Delta$ is Gorenstein* of dimension $d-1$, so that

$$
\operatorname{dim}(K[\Delta])=\operatorname{reg}(K[\Delta])=d .
$$


Let $\sigma$ be a $(d-3)$-dimensional face of $\Delta$. Since $C=\mathrm{lk}_{\Delta} \sigma$ is a 1-dimensional flag Gorenstein* complex, it must be a $k$-cycle for some $k \geq 4$. Using flagness again, it follows that $C$ is an induced $k$-cycle of $\Delta$.

Let $A$ be the average number of facets of $\Delta$ containing a given $(d-3)$-dimensional face. Since any facet of $\Delta$ contains exactly $\left(\begin{array}{l}d \\ 2\end{array}\right)$ faces of dimension $d-3$, the average number is

$$
A=\frac{f_{d-1}}{f_{d-3}}\left(\begin{array}{l}
d \\
2
\end{array}\right) .
$$

Since $K[\Delta]$ is Gorenstein of regularity $d$, the Dehn-Sommerville equations $h_{i}=h_{d-i}$ hold for $i=0, \ldots, \delta:=\lfloor d / 2\rfloor$. Let

$$
\widehat{h_{i}}:= \begin{cases}h_{i} & \text { if } i<d / 2 \\ h_{i} / 2 & \text { if } i=d / 2\end{cases}
$$

From the relation between $f$ - and $h$-vector $\left(f_{j-1}=\sum_{i=0}^{j}\left(\begin{array}{c}d-i \\ j-i\end{array}\right) h_{i}\right.$ for $\left.j=0, \ldots, d\right)$ we get

$$
f_{d-1}=2 \cdot \sum_{i=0}^{\delta} \widehat{h}_{i} \quad \text { and } \quad f_{d-3}=\sum_{i=0}^{\delta}\left(\left(\begin{array}{c}
d-i \\
2
\end{array}\right)+\left(\begin{array}{l}
i \\
2
\end{array}\right)\right) \widehat{h_{i}} .
$$

Since $\left(\begin{array}{c}d-i \\ 2\end{array}\right)+\left(\begin{array}{c}i \\ 2\end{array}\right)<\left(\begin{array}{c}d-(i-1) \\ 2\end{array}\right)+\left(\begin{array}{c}i-1 \\ 2\end{array}\right)$ for $i=1, \ldots, \delta$ we get $f_{d-3}>\left(\left(\begin{array}{c}d-\delta \\ 2\end{array}\right)+\left(\begin{array}{l}\delta \\ 2\end{array}\right)\right) \sum_{i=0}^{\delta} \widehat{h_{i}}$. Therefore

$$
A<\frac{2 \cdot\left(\begin{array}{l}
d \\
2
\end{array}\right)}{\left(\begin{array}{c}
d-\delta \\
2
\end{array}\right)+\left(\begin{array}{l}
\delta \\
2
\end{array}\right)} .
$$

The right hand side of the above inequality evaluates as

$$
A< \begin{cases}\frac{4(d-1)}{d-2} & \text { if } d \text { is even } \\ \frac{4 d}{d-1} & \text { if } d \text { is odd }\end{cases}
$$

In particular, if $d>4$, then $A<5$, so there exists a $(d-3)$-dimensional face $\sigma$ of $\Delta$ such that $\mathrm{lk}_{\Delta} \sigma$ is a 4-cycle. If $d>2$, then $A<6$, so there exists a $(d-3)$-dimensional face $\sigma$ of $\Delta$ such that $\mathrm{lk}_{\Delta} \sigma$ is a 5 -cycle. By the first paragraph of this proof, such cycles are induced cycles of $\Delta$, so we get a contradiction to $S / I$ satisfying, respectively, $N_{2}$ or $N_{3}$.

Among edge ideals with linear syzygies, the example with highest regularity in DHS13. achieved the value four. This limitation may be due to the fact that the natural strategy to produce such examples is to construct a flag-no-square triangulation of a $d$-sphere for high $d$. However, by Theorem 4, such triangulations do not exist whenever $d>3$. Even more, Theorem 4 implies that, in order to find examples of unbounded regularity, one has to leave the world of manifolds too:

Remark 5. Let $\Delta$ be a flag-no-square triangulation of a (homology) $d$-manifold. Then $d \leq 4$. This follows immediately from Theorem 4 because $\mathrm{lk}_{\Delta} v$ is a flag-no-square triangulation of a (homology) $(d-1)$-sphere. 
Dropping the insistence on manifolds, however, it is possible to find edge ideals with linear syzygies and arbitrarily high regularity. We found them-somewhat surprisingly - related to a question of Gromov on the existence of hyperbolic Coxeter groups with arbitrarily high virtual cohomological dimension. The question was answered positively by Januszkiewicz and Świątkowski in [JŚ03. The interesting fact for our purposes is that, to answer Gromov's question, they built a flag-no-square closed orientable pseudomanifold of dimension $r$ for any positive integer $r$. These complexes are denoted $L_{r}$ (see [JŚ03, Section 6]).

A simplicial complex $\Delta$ is a closed pseudomanifold if it is strongly connected (in particular pure) and any codimension 1 face is contained in exactly two facets. A closed pseudomanifold is called orientable when for any codimension 1 face $F$, if $F \cup\{i\}$ and $F \cup\{j\}$ are the two facets containing it then $|\{k \in F: k<i\}|+|\{k \in F: k<j\}|$ is odd.

Theorem 6. For any integer $r \geq 1$, there exists a graph $G_{r}$ on $n(r)$ vertices such that $S / I\left(G_{r}\right)$ satisfies $N_{2}$, where $S=K\left[x_{1}, \ldots, x_{n(r)}\right]$, and

$$
\operatorname{reg}\left(S / I\left(G_{r}\right)\right)=r .
$$

Proof. Let $G_{r}$ be the complement of the 1-skeleton of $L_{r-1}$, so that $L_{r-1}=\operatorname{In}\left(G_{r}\right)$. Then $S / I\left(G_{r}\right)$ satisfies $N_{2}$. Furthermore, because $L_{r-1}$ is a closed orientable pseudomanifold of dimension $r-1$, it is straightforward to check that

$$
\sum_{F \text { facet of } L_{r-1}} F
$$

is a top-dimensional cycle. In particular

$$
\widetilde{H}_{r-1}\left(L_{r-1} ; K\right) \neq 0 .
$$

By Hochster's formula [MS05, Corollary 5.12]), $\operatorname{reg}\left(K\left[L_{r-1}\right]\right)=r$.

As noted in [JS03], the number $n(r)$ in the above theorem is huge, growing much more quickly than exponential in $r$. Consequently the family $\left\{L_{r}\right\}_{r}$ is not suitable to show that Theorem 2 is (asymptotically) sharp. In [JŚ03] it was also observed that any family of flagno-square pseudomanifolds $\Delta$ of dimension $d$ is forced to have a huge number of vertices.

In the remainder of this work we quantify their result and extend its proof to flag-no-square complexes with no free codimension 1 faces, i.e. codimension 1 faces contained in only one facet (Theorem 8). To this end we prove that the number of vertices of such a simplicial complex is at least doubly exponential in the dimension.

Lemma 7. For any integer $k \geq 3$,

$$
\prod_{i=0}^{k-3}(k-i)^{2^{i}}<12^{2^{k-3}} .
$$

Proof. This is a routine computation using the inequality $(i-1)(i+1)<i^{2}$ several times.

Theorem 8. Let $\Delta$ be a d-dimensional flag-no-square simplicial complex with no free $(d-1)$ faces. Then, if $\left(f_{-1}, f_{0}, \ldots, f_{d}\right)$ is the $f$-vector of $\Delta$,

$$
f_{d}>(25 / 12)^{2^{d-2}} \quad \text { and } \quad f_{0}>(25 / 12)^{2^{d-3}} .
$$


Proof. Let $v_{d}$ and $s_{d}$ denote respectively the minimal $f_{0}(\Delta)$ and $f_{d}(\Delta)$ attained in the class of simplicial complexes in the statement. Let $\Delta$ be such a complex. The link $\mathrm{lk}_{\Delta} v$ of any vertex $v \in \Delta$ is a $(d-1)$-dimensional flag-no-square simplicial complex with no free $(d-2)$-faces. By double counting, we find

$$
f_{d}(\Delta)=\frac{1}{d+1} \sum_{v \in \Delta} f_{d-1}\left(\mathrm{lk}_{\Delta} v\right)
$$

Therefore

$$
f_{d}(\Delta) \geq \frac{f_{0}(\Delta) \cdot s_{d-1}}{d+1} .
$$

Fix a vertex $v \in \Delta$. Any facet of $\mathrm{lk}_{\Delta} v$ is a $(d-1)$-face of $\Delta$. Since $\Delta$ has no free $(d-1)$-faces, to any facet of $\mathrm{lk}_{\Delta} v$, we can associate a vertex of $\Delta$ which does not belong to the star of $v$. In other words we defined a function

$$
\phi: \mathcal{F}_{d-1}\left(\mathrm{lk}_{\Delta} v\right) \longrightarrow \mathcal{F}_{0}(\Delta) \backslash \mathcal{F}_{0}\left(\operatorname{star}_{\Delta} v\right) .
$$

Because of the no-square condition, and since $\mathrm{lk}_{\Delta} v$ is an induced subcomplex of $\Delta$, one can check that $\phi$ is injective and thus

$$
f_{0}(\Delta) \geq s_{d-1}+v_{d-1}+1 .
$$

In particular, putting together the above inequalities we get

$$
s_{d}>\frac{s_{d-1}^{2}}{d+1} \quad v_{d}>s_{d-1} .
$$

Since $s_{1}=5$, we find

$$
f_{d}(\Delta)>\frac{5^{2^{d-1}}}{\prod_{i=0}^{d-2}(d+1-i)^{2^{i}}} .
$$

Finally, by Lemma 7 .

$$
f_{d}(\Delta)>\frac{5^{2^{d-1}}}{12^{2^{d-2}}}=(25 / 12)^{2^{d-2}}
$$

Remark 9. Unfortunately the doubly exponential bound in Theorem 8 can not be easily extended to arbitrary flag-no-square simplicial complexes (replacing $d$ with the top degree in which the homology does not vanish). While it is always possible to get rid of the free faces by collapses, indeed, this operation does not preserve flagness. For a general flag-nosquare simplicial complex, the exponential bound found by Dao, Huneke, and Schweig is to our knowledge the best possible.

Remark 10. If a flag-no-square simplicial complex of dimension 2 has a free 1-face $e \subset F$, where $F$ is the only 2 -face containing $e$, then if we collapse the pair $(e, F)$ we still get a flagno-square simplicial complex (this is a peculiarity of the dimension 2 case). This observation, together with the proof of Theorem 8 , yields the following: Let $I(G) \subseteq S=K\left[x_{1}, \ldots, x_{n}\right]$ be an edge ideal such that $\operatorname{dim}(S / I(G))=\operatorname{reg}(S / I(G))=3$. If $S / I(G)$ satisfies $N_{2}$, then $n \geq 12$. If $n=12$, then $\operatorname{In}(G)$ is the boundary of the icosahedron. 


\section{REFERENCES}

[ACI13] Luchezar L Avramov, Aldo Conca, and Srikanth B Iyengar, Subadditivity of syzygies of koszul algebras, Mathematische Annalen (2013), to appear.

[BM93] Dave Bayer and David Mumford, What can be computed in algebraic geometry?, Computational algebraic geometry and commutative algebra (Cortona 1991), Sympos. Math., vol. XXXIV, Cambridge University Press, 1993, pp. 1-48.

[BS88] David Bayer and Michael Stillman, On the complexity of computing syzygies, Journal of Symbolic Computation 6 (1988), no. 2, 135-147.

[Dav08] Michael Davis, The geometry and topology of coxeter groups, vol. 32, Princeton University Press, 2008.

[DHS13] Hailong Dao, Craig Huneke, and Jay Schweig, Bounds on the regularity and projective dimension of ideals associated to graphs, Journal of Algebraic Combinatorics 38 (2013), no. 1, 37-55.

[EG84] David Eisenbud and Shiro Goto, Linear free resolutions and minimal multiplicity, Journal of Algebra 88 (1984), no. 1, 89-133.

[JŚ03] Tadeusz Januszkiewicz and Jacek Świątkowski, Hyperbolic coxeter groups of large dimension, Commentarii Mathematici Helvetici 78 (2003), no. 3, 555-583.

[MM82] Ernst W. Mayr and Albert A. Meyer, The complexity of the word problems for commutative semigroups and polynomial ideals, Advances in Mathematics 46 (1982), no. 3, 305-329.

[MS05] Ezra Miller and Bernd Sturmfels, Combinatorial commutative algebra, GTM, vol. 227, Springer, Berlin, 2005.

[Sta77] Richard P. Stanley, Cohen-Macaulay complexes, in Higher Combinatorics 31 (1977), 51-62.

Mathematisches Institut, Freie Universität Berlin, Arnimallee 3, 14195 Berlin, Germany

E-mail address: aconstant@math.fu-berlin.de

$U R L:$ http://userpage.fu-berlin.de/aconstant/Main.html

Fakultät für Mathematik, OvgU Magdeburg, Universitätsplatz 2, 39106 Magdeburg, GerMANY

E-mail address: thomas.kahle@ovgu.de

$U R L:$ http://www.thomas-kahle.de

Dipartimento di Matematica, Università di Genova, Via Dodecaneso 35, Genova 16146, Italy

E-mail address: varbaro@dima.unige.it

$U R L:$ http://www.dima.unige.it/ varbaro/ 